\title{
Comparative study of normal and branched alkane monolayer films adsorbed on a solid surface. I. Structure
}

\author{
A. D. Enevoldsen and F. Y. Hansen ${ }^{\text {a) }}$ \\ Department of Chemistry, Technical University of Denmark, IK 207 DTU, DK-2800 Lyngby, Denmark
}

\author{
A. Diama, L. Criswell, and H. Taub \\ Department of Physics and Astronomy, University of Missouri-Columbia, Columbia, Missouri 65211 \\ and The University of Missouri Research Reactor Center, University of Missouri-Columbia, Columbia, \\ Missouri 65211
}

(Received 21 September 2006; accepted 9 January 2007; published online 13 March 2007)

\begin{abstract}
The structure of a monolayer film of the branched alkane squalane $\left(\mathrm{C}_{30} \mathrm{H}_{62}\right)$ adsorbed on graphite has been studied by neutron diffraction and molecular dynamics (MD) simulations and compared with a similar study of the $n$-alkane tetracosane $\left(n-\mathrm{C}_{24} \mathrm{H}_{52}\right)$. Both molecules have 24 carbon atoms along their backbone and squalane has, in addition, six methyl side groups. Upon adsorption, there are significant differences as well as similarities in the behavior of these molecular films. Both molecules form ordered structures at low temperatures; however, while the melting point of the two-dimensional (2D) tetracosane film is roughly the same as the bulk melting point, the surface strongly stabilizes the $2 \mathrm{D}$ squalane film such that its melting point is $91 \mathrm{~K}$ above its value in bulk. Therefore, squalane, like tetracosane, will be a poor lubricant in those nanoscale devices that require a fluid lubricant at room temperature. The neutron diffraction data show that the translational order in the squalane monolayer is significantly less than in the tetracosane monolayer. The authors' MD simulations suggest that this is caused by a distortion of the squalane molecules upon adsorption on the graphite surface. When the molecules are allowed to relax on the surface, they distort such that all six methyl groups point away from the surface. This results in a reduction in the monolayer's translational order characterized by a decrease in its coherence length and hence a broadening of the diffraction peaks. The MD simulations also show that the melting mechanism in the squalane monolayer is the same footprint reduction mechanism found in the tetracosane monolayer, where a chain melting drives the lattice melting. (C) 2007 American Institute of Physics.
\end{abstract}

[DOI: 10.1063/1.2464091]

\section{INTRODUCTION}

The main constituents of commercial lubricants are intermediate-length alkanes $\mathrm{C}_{n} \mathrm{H}_{2 n+2}$ with the number of carbon atoms $n$ in the range of 15-40. The vapor pressure of these alkanes at ambient temperature is low so that the evaporation rate is very small. Lubricants are characterized by their pour point, viscosity index, and viscosity. The pour point is the lowest temperature at which the lubricant can be used. Below the pour point, the material forms wax crystals, resulting in an increase in its viscosity and poorer lubricating properties. The viscosity index is a measure of the sensitivity of the viscosity to temperature changes, with a high index corresponding to a low sensitivity. Therefore, a good lubricant is characterized by low viscosity, a high viscosity index, and low pour point. 1,2

Among alkanes, it has been found that branched alkanes are superior to $n$-alkanes as lubricants at ambient temperatures, primarily due to a lower pour point and viscosity. This is illustrated in Table $\mathrm{I}^{1}$, where we compare data for the $n$-alkane tetracosane $\mathrm{C}_{24} \mathrm{H}_{52}(\mathrm{C} 24)$ and squalane $\left(\mathrm{C}_{30} \mathrm{H}_{62}\right.$ or 2,6,10,15,19,23-hexamethyltetracosane). Like C24, a

${ }^{a)}$ Electronic mail: flemming @kemi.dtu.dk squalane molecule has 24 carbon atoms in its backbone and, in addition, six symmetrically placed methyl groups along its length, as shown in Fig. 1. In Table I, we see that C24 has a pour point above room temperature, reflecting its high bulk melting point compared to squalane. The squalane molecule's branching in the form of six methyl side groups dramatically affects its bulk melting point, dropping it $88 \mathrm{~K}$ below that of $\mathrm{C} 24$.

It is not obvious, however, that bulk properties alone determine the lubrication characteristics of a material. Usually, a lubricant is distributed in a very thin film between two solid surfaces that undergo relative motion; in some nanoscale electromechanical devices, this film thickness may approach a few molecular layers. In such cases, the lubricant's properties may differ significantly from those in bulk and, in

TABLE I. Characteristic parameters for lubricants (from Ref. 1).

\begin{tabular}{ccc}
\hline \hline & Tetracosane & Squalane \\
\hline Pour point $(\mathrm{K})$ & 324 & 294 \\
Viscosity index & 175 & 117 \\
Bulk melting point $(\mathrm{K})$ & 322 & 234 \\
Viscosity at 313 K $\left(\mathrm{mm}^{2} \mathrm{~s}^{-1}\right)$ & $\ldots$ & 19.3 \\
Viscosity at 373 K $\left(\mathrm{mm}^{2} \mathrm{~s}^{-1}\right)$ & 8.7 & 4.1 \\
\hline \hline
\end{tabular}


particular, the lubricant film could become solid rather than liquid. This could affect the frictional properties of the film, although a solid film could still potentially protect neighboring surfaces from wear prior to complete squeeze-out. ${ }^{3}$

At the molecular level, very little is known about how a film's structure, dynamics, and energy dissipation relate to the lubricating properties of a substance. Such knowledge may be important in designing new lubricants for nanoscale devices. Gupta et al. have conducted nonequilibrium molecular dynamics simulations, in which a few layers of C24 or squalane molecules are placed between solid walls that move with respect to each other, thereby setting up a shear flow. ${ }^{4-6}$ These authors found that squalane actually was a poorer lubricant and attributed it to the presence of the methyl side groups that prevented the molecules from organizing into smooth layers as did C24.

In this paper, we study the structure of molecular monolayers of $\mathrm{C} 24$ and squalane as a function of temperature by neutron diffraction and molecular dynamics (MD) simulations. In both the experiment and the simulations, the films are adsorbed on a graphite basal-plane surface. This choice is dictated by the requirement of a large surface area for the neutron diffraction experiments, since neutrons are a weakly scattering probe. Exfoliated graphite substrates that offer a large surface and an exceptionally homogeneous surface area are available. Graphite is also well suited to MD simulations because the molecule-substrate interaction is well characterized by empirical pairwise atomic potentials.

Although we consider only a single film/solid interface rather than a lubricant confined between two solid surfaces, we believe that a comprehensive investigation of the structure and dynamics of these adsorbed monolayers is a useful initial step toward understanding lubrication phenomena at the molecular level. For example, it is of interest to determine whether the intermolecular and molecule-substrate van der Waals interactions result in a distortion of flexible molecules upon adsorption as well as determine the molecular orientation and the nature of the translational order in the monolayer. These structural properties are helpful in describing the initial condition of lubricant films at large separations of their confining surfaces. One is then in a better position to investigate the squeeze-out behavior of these films as the separation of the confining surfaces is reduced. ${ }^{3,7,8}$

Because we are dealing with lubricant films that interact with a substrate by van der Waals forces, we do not believe that it is a serious restriction to limit our investigation to adsorption on graphite. As discussed elsewhere, in all known cases of alkanes interacting with solid surfaces via van der Waals forces, the molecules immediately adjacent to the surface are aligned with their long axis parallel to the interface. ${ }^{9}$ The binding energy of the alkane molecule varies, of course, among different substrates and alkanes of different lengths (see, e.g., Ref. 10), but we believe that the squeeze-out behavior of films of intermediate-length alkanes will be largely determined by this adsorption geometry. ${ }^{3}$

In comparing the structure of molecular monolayers of $\mathrm{C} 24$ and squalane as a function of temperature, we focus on the following questions: (a) What is the effect of branching on the monolayer structure? (b) Do the monolayer melting points of the two alkanes differ by as much as their bulk melting points? (c) Are the melting mechanisms the same for the two films? In the following paper, ${ }^{11}$ we use quasielastic neutron scattering and MD simulations to study the dynamics of such layers.

The organization of the paper is as follows. In Sec. II, we describe the experimental technique and in Sec. III the simulations. In Sec. IV, we describe and compare the results for the $n$-alkane C24 and the branched alkane squalane. Finally, in Sec. V, we present a summary of our results and conclusions.

\section{EXPERIMENTS}

We have used neutron diffraction to compare the monolayer structure of C24 and squalane adsorbed on a graphite basal-plane surface. Since neutrons interact weakly with the sample, a relatively large sample is required in order to obtain reasonable diffraction intensities.

The sample preparation technique has been described in detail elsewhere. ${ }^{12}$ In brief, the substrates were recompressed exfoliated graphite (Grafoil), whose surface area was calibrated from a nitrogen vapor pressure adsorption isotherm at $77 \mathrm{~K}$. The C24 and squalane adsorbates were 98\% deuterated to avoid the large incoherent scattering from hydrogen. Vapor deposition of the C24 monolayer was accomplished by heating the $\mathrm{C} 24$ charge and cleaned Grafoil substrate in an evacuated stainless steel cell at $280{ }^{\circ} \mathrm{C}$ for $48 \mathrm{~h} .{ }^{13}$ The squalane monolayer was similarly prepared by heating the liquid squalane charge in the same manner at $230{ }^{\circ} \mathrm{C}$ for $32 \mathrm{~h} .{ }^{14}$ In both cases, Grafoil background diffraction patterns were measured at room temperature before adsorption of the alkanes.

The neutron diffraction patterns were measured using the two-axis diffractometer equipped with a five-counter multidetector data acquisition system located at the $\mathrm{C}$ port of the Missouri University Research Reactor. The measurements were done in a transmission geometry, as in previous experiments, ${ }^{15}$ and used a wavelength of $4.35 \AA$, which is appropriate to the relatively large monolayer unit cell of the alkane molecules. The low-temperature diffraction patterns for squalane $(T<300 \mathrm{~K})$ (Ref. 16) have been published previously, while the high-temperature data for squalane $(T>300 \mathrm{~K})$ are new. The diffraction measurements on the C24 monolayers will be published separately. ${ }^{17}$

\section{MOLECULAR DYNAMICS SIMULATIONS}

\section{A. Model of molecule}

In the molecular dynamics simulations, we use the united atom model for the alkane molecules, where the methylene and methyl groups are represented by pseudoatoms having the proper atomic weight located at the carbon atom positions. Figure 1(a) shows a top-down view of the united atom model of a free squalane molecule in its ground state. There are 24 carbon atoms forming a zigzag pattern in the plane of the paper that comprise the molecular backbone. Only bonds between pseudoatoms are shown. There are six bonds labeled $\mathrm{S} 1-\mathrm{S} 6$ from carbon atoms in the backbone to the methyl side groups. 
(a)

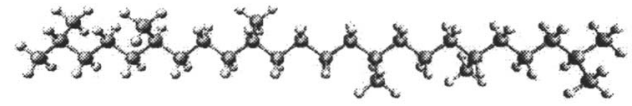

(b)

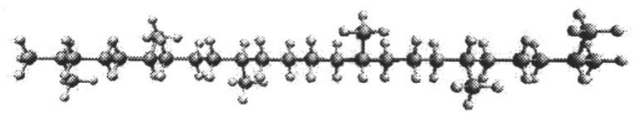

FIG. 1. (a) Top view of a squalane molecule in the ground state with the plane of the carbon atoms in the backbone parallel to the plane of the paper. (b) Side view of the squalane molecule with the plane of the carbon backbone perpendicular to the plane of the paper. The methyl side groups are seen to be out of the plane of the backbone. The large and small circles are carbon and hydrogen atoms, respectively.

The interactions between methylene and methyl groups on different molecules are represented by the same effective Lennard-Jones potential, irrespective of which of the two groups are involved. In simulations of both this monolayer and bulk systems, it is essential to include the molecular flexibility associated with the bond angles and dihedral torsion angles; however, the bond stretch modes are unimportant and have therefore been frozen. The potential parameters are the same as we have used previously. ${ }^{18-21}$

The backbone of the squalane molecule contains $21 \mathrm{di}$ hedral torsion angles numbered from 1 to 21 from the left. As illustrated in Fig. 2, each of the four side groups, $\mathrm{Si}(i=2,5)$, can torsion about either of the two carbon-carbon bonds in the backbone to which the side groups are attached. The end methyl groups, S1 and S6, can only torsion about the next-to-the-last bond at each end of the backbone. In the molecular ground state, the dihedral torsion angles about the carbon-carbon bonds along the backbone (angles 1-21) are in the trans conformation, whereas all dihedral torsion angles involving the side groups are in a gauche conformation. There are two gauche conformations, that we label as left and right, corresponding to a rotation about the carboncarbon bond of $\pm 120^{\circ}$, respectively, from the trans conformation. More precisely, given the position vectors of the four atoms $\left(\mathbf{r}_{A}, \mathbf{r}_{B}, \mathbf{r}_{C}, \mathbf{r}_{D}\right)$ involved in the definition of the dihedral torsion angle about the $B C$ bond $\left(\mathbf{r}_{B}-\mathbf{r}_{C}\right)$, we arbitrarily define a left and right gauche conformation according to the following relations:

$$
\begin{aligned}
& {\left[\left(\mathbf{r}_{A}-\mathbf{r}_{B}\right) \times\left(\mathbf{r}_{C}-\mathbf{r}_{D}\right)\right] \cdot\left(\mathbf{r}_{D}-\mathbf{r}_{C}\right)<0, \text { left gauche, }} \\
& {\left[\left(\mathbf{r}_{A}-\mathbf{r}_{B}\right) \times\left(\mathbf{r}_{C}-\mathbf{r}_{D}\right)\right] \cdot\left(\mathbf{r}_{D}-\mathbf{r}_{C}\right)>0, \text { right gauche. }}
\end{aligned}
$$

With the molecule in the ground state, we see that one of the pair of dihedral torsion angles associated with each of the side groups, $\mathrm{S} i(i=2,5)$, will be in a right gauche conformation, while the other will be in a left gauche conformation.

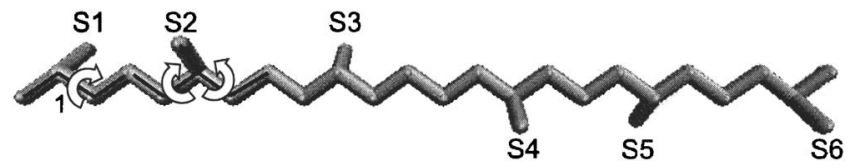

FIG. 2. Top view of the pseudoatom model for an adsorbed squalane molecule. Only the bonds between pseudoatoms are shown, and the side groups are labeled S1-S6. Illustration of the pair of torsion angles associated with each of the side groups $\mathrm{S} i$, for $i=2-5$, and the single torsion angle associated with the two side groups, S1 and S6.

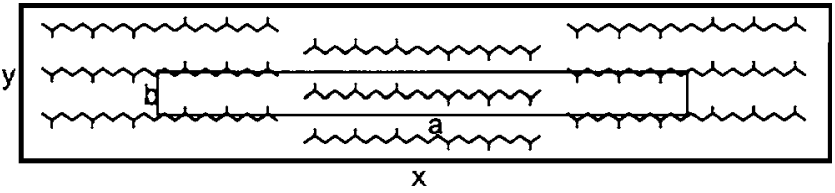

FIG. 3. Sketch of the proposed unit cell of the squalane monolayer adsorbed on a graphite basal-plane surface. It is a rectangular-centered structure containing two molecules with lattice constants $a$ and $b$.

The gauche conformation associated with the end groups $\mathrm{S} 1$ and S6 can be either a left or a right form.

\section{B. Simulation box}

The first step in a MD simulation is the choice of the simulation cell and the number of molecules to be included in the simulations. The choice of simulation box should satisfy three requirements: (a) give an isotropic stress tensor with $P_{x x} \sim P_{y y}$ where $P_{x x}$ is the $x x$ component of the stress tensor and $P_{y y}$ the $y y$ component, (b) satisfy the translational symmetry of the graphite basal-plane lattice, and (c) give alkane monolayer lattice vectors $a_{\text {latt }}$ and $b_{\text {latt }}$ in good agreement with experimental determined lattice vectors.

With the $x$ axis taken along the direction from a center of one carbon hexagon to an adjacent one in the graphite basal plane, the conditions in (b) are satisfied by the relations ${ }^{19}$

$$
\begin{aligned}
& x_{\text {sim }}=a_{\text {latt }} n_{a}=2.46 \times l_{x}, \\
& y_{\text {sim }}=b_{\text {latt }} n_{b}=2 \times 2.1304 \times l_{y} .
\end{aligned}
$$

The carbon-carbon distance in the graphite basal-plane layer equals $1.42 \AA$. These equations ensure that the simulation box dimensions in the $x$ and $y$ directions, $x_{\text {sim }}$ and $y_{\text {sim }}$, respectively, are a multiple of the graphite lattice constant, as expressed by the integers $l_{x}$ and $l_{y}$ as well as being a multiple of the alkane lattice constants as expressed by the integers $n_{a}$ and $n_{b}$.

It is natural to be guided by the low-temperature neutron diffraction results that give the experimental lattice constants $a$ and $b$ of the alkane system. As will be discussed in the next section, the experiments are consistent with the squalane monolayer, like the $\mathrm{C} 24$ monolayer, ${ }^{17}$ crystallizing in a rectangular-centered structure with two molecules in the unit cell, as sketched in Fig. 3. ${ }^{15}$ However, it will not necessarily be correct to use the experimental values of the lattice constants in the simulation directly, because the model interactions assumed may not be perfect and give precisely the experimental values. If one uses the experimental values for the lattice constants, one may find unphysical values of other properties of the system. For example, the pressure may be negative or very large. We have no experimental values of the pressure in the system, but we may assume that it is near zero at low temperatures. We use that criterion together with the intermolecular and molecule-surface interaction energies as a guide to set up the simulation self-consistently. That is, we seek a setup where the pressure tensor is isotropic with zero or near-zero pressure components and where we are near a minimum in the intermolecular and moleculesubstrate potential energies. 
TABLE II. Determination of the initial parameters for the squalane simulations at $86 \mathrm{~K}$.

\begin{tabular}{|c|c|c|c|c|c|c|c|c|}
\hline$n_{a}$ & $\begin{array}{l}a_{\text {latt }} \\
(\AA)\end{array}$ & $n_{b}$ & $\begin{array}{l}b_{\text {latt }} \\
(\AA)\end{array}$ & $\begin{array}{c}\text { Number of } \\
\text { molecules }\end{array}$ & $\begin{array}{l}\text { Pressure } \\
(\mathrm{Pa})\end{array}$ & $\begin{array}{c}\text { Intermolecular } \\
\text { energy } \\
(\mathrm{K})\end{array}$ & $\begin{array}{c}\text { Molecule/ } \\
\text { substrate } \\
\text { energy } \\
\text { (K) }\end{array}$ & $\begin{array}{l}\text { Distance } \\
\text { from } \\
\text { surface } \\
(\AA)\end{array}$ \\
\hline 2 & 63.96 & 10 & 5.113 & 40 & 0.0186 & -8842 & -25685 & 3.99 \\
\hline 2 & 63.96 & 9 & 5.208 & 36 & 0.019 & -8531 & -26170 & 3.94 \\
\hline 2 & 63.96 & 8 & 5.326 & 32 & -0.0109 & -8276 & -26523 & 3.91 \\
\hline 2 & 63.96 & 11 & 5.423 & 44 & -0.0265 & -8172 & -26511 & 3.92 \\
\hline
\end{tabular}

As in the simulations of the $\mathrm{C} 24$ monolayer, ${ }^{17}$ we made a series of simulations at low temperature, varying the alkane lattice constants and the number of molecules in the simulation cell such that Eqs. (2) were satisfied. In the case of the C24 monolayer, we used a temperature of $20 \mathrm{~K}$. However, since the squalane molecule turns out to be distorted by the presence of the graphite surface, as described below, we conducted the low-temperature squalane monolayer simulations at $86 \mathrm{~K}$ to speed up the approach to an equilibrium structure. In Table II, we show an example of this procedure.

We see that the pressure changes from being positive for the parameters in the second line of the table to being negative for the parameters in the third line. Ideally, we should choose a simulation box length corresponding to parameters between those in lines 2 and 3 of the table, so the pressure is close to zero, but we cannot do that without violating the constraints in Eqs. (2). When we look at the moleculesurface energy and the molecular height above the surface, we see that the height is smallest for the parameters in line 3 and that the molecule-substrate energy is at a minimum. This is what we would expect at low temperatures for the thermodynamically stable state; we have, therefore, chosen to do the simulations with the parameters in line 3 of Table II. Note that the lowest molecule-substrate energy is obtained at the cost of the intermolecular energy. When we compress the system and increase the pressure, the molecules rotate about their long axis to accommodate the pressure increase and gain intermolecular energy at the cost of molecule-substrate energy. The value of the lattice constant $a_{\text {latt }}=64 \AA$ obtained in the simulations is equal, within experimental uncertainty, to that determined for a $\mathrm{C} 24$ monolayer ${ }^{17}$ and which is the value we assume (see below) for the squalane monolayer at low temperature. The lattice constant $b_{\text {latt }}=5.33 \AA$ obtained in the simulation is about $5 \%-6 \%$ larger than the experimental value of $5.03 \AA$ at a temperature of $86 \mathrm{~K},{ }^{16}$ as discussed below. This level of agreement between simulation and experiment for $b_{\text {latt }}$ is comparable to that obtained for a $\mathrm{C} 24$ monolayer. ${ }^{17}$

\section{RESULTS}

In the low-temperature diffraction patterns of the $\mathrm{C} 24$ monolayer, we were able to resolve four Bragg peaks in the $(h 0)$ series at small wave vector transfer $q$ as well as the (11) peak. This allowed us to determine the monolayer unit cell as rectangular centered and containing two molecules with lattice constants $a=65 \AA$ and $b=4.26 \AA . .^{16,17}$
In contrast, it was only possible to resolve one Bragg peak of the squalane monolayer due to the short coherence length of the film. Because the lengths of the C24 and squalane molecules are identical in their ground state, we assumed a similar structure for the squalane monolayer and used the $a$ lattice constant determined for the C24 monolayer. Indexing the single peak in the squalane monolayer diffraction pattern as the (11) reflection, we then obtained $b=5.03 \AA$ by fitting a Warren line shape to the peak in the pattern at $86 \mathrm{~K}$ in Fig. 4(a). Although a Warren line shape is appropriate for a two-dimensional (2D) polycrystalline system, ${ }^{15}$ we found it difficult to obtain reliable results using it to fit the very broad Bragg peak occurring in the diffraction patterns at temperatures higher than $86 \mathrm{~K}$. Therefore, we fit the peak to a Gaussian line shape and introduced a linear background in order to reproduce the peak asymmetry. In Fig. 4, the solid curves are the best fits obtained in this way and the open circles are the data points. We note that at the lowest temperature of $86 \mathrm{~K}$, a fit to a Gaussian line shape yields a slightly smaller lattice constant $b=4.95 \AA$ compared to the value of $5.03 \AA$ obtained using a Warren line shape. Although we believe the peak at $86 \mathrm{~K}$ to be sufficiently narrow for the Warren fit to give a more accurate lattice constant, we have used a Gaussian fit throughout Fig. 4 in order to show more easily the temperature dependence of the $b$ lattice constant as in Fig. 5.

In the case of the C24 monolayer, ${ }^{16,17}$ the (11) Bragg peak was narrow enough to fit it to a Warren line shape over a broader range of temperatures. In order to be able to compare the coherence lengths in the two monolayers, it is necessary to determine a scale factor relating the coherence lengths obtained with the two different line shapes. To do this, we required the fits of the diffraction peak of the squalane monolayer at $86 \mathrm{~K}$ to a Gaussian and a Warren line shape to give the same coherence length. This yielded the empirical relation for the coherence length, $L_{b}$ $=1.31(2 \pi / \Delta q)$, where $\Delta q$ is the full width at half maximum of the Gaussian fit to the diffraction peak. $L_{b}$ is plotted as a function of temperature in Fig. 5.

\section{A. Low temperature}

When we compare the low-temperature $(T<300 \mathrm{~K})$ structure for the squalane and $\mathrm{C} 24$ monolayers, we notice three features. First, we find that the $b$ lattice constant of the C24 monolayer is $4.26 \AA$ at low temperature, while that for squalane is larger with a value of $5.03 \AA$. This was expected, and it reflects the presence of the methyl side groups in the 

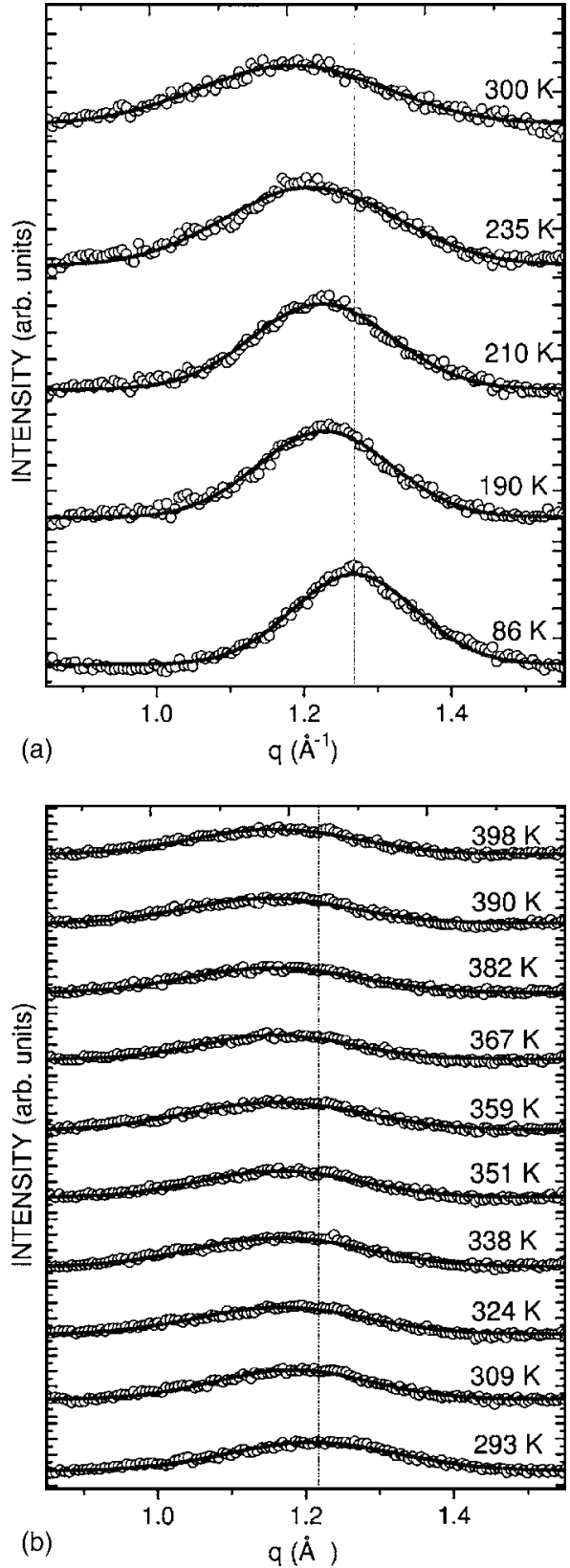

FIG. 4. Neutron diffraction patterns from a 0.96-layer deuterated squalane film adsorbed on Grafoil: (a) below room temperature (from Ref. 16) and (b) above room temperature. The solid curves are fits to the patterns using a Gaussian line shape, as described in the text. Error bars are not shown for clarity but are commensurate with the indicated scatter in the data points.

squalane molecule. The lattice constant of the C24 monolayer is commensurate with the graphite basal-plane surface, indicating that the corrugation of the molecule-surface holding potential is important for the formation of the crystalline monolayer structure. On the other hand, the squalane monolayer $b$ lattice constant is incommensurate with the graphite surface so the effect of the corrugation in the holding potential is less certain.

Second, there seems to be a phase transition below a temperature of $230 \mathrm{~K}$ in both monolayers, as seen by a marked decrease in the coherence length on heating and a change in the coefficient of thermal expansion in the $\mathbf{b}$ direction. The drop in the coherence length implies a less or-
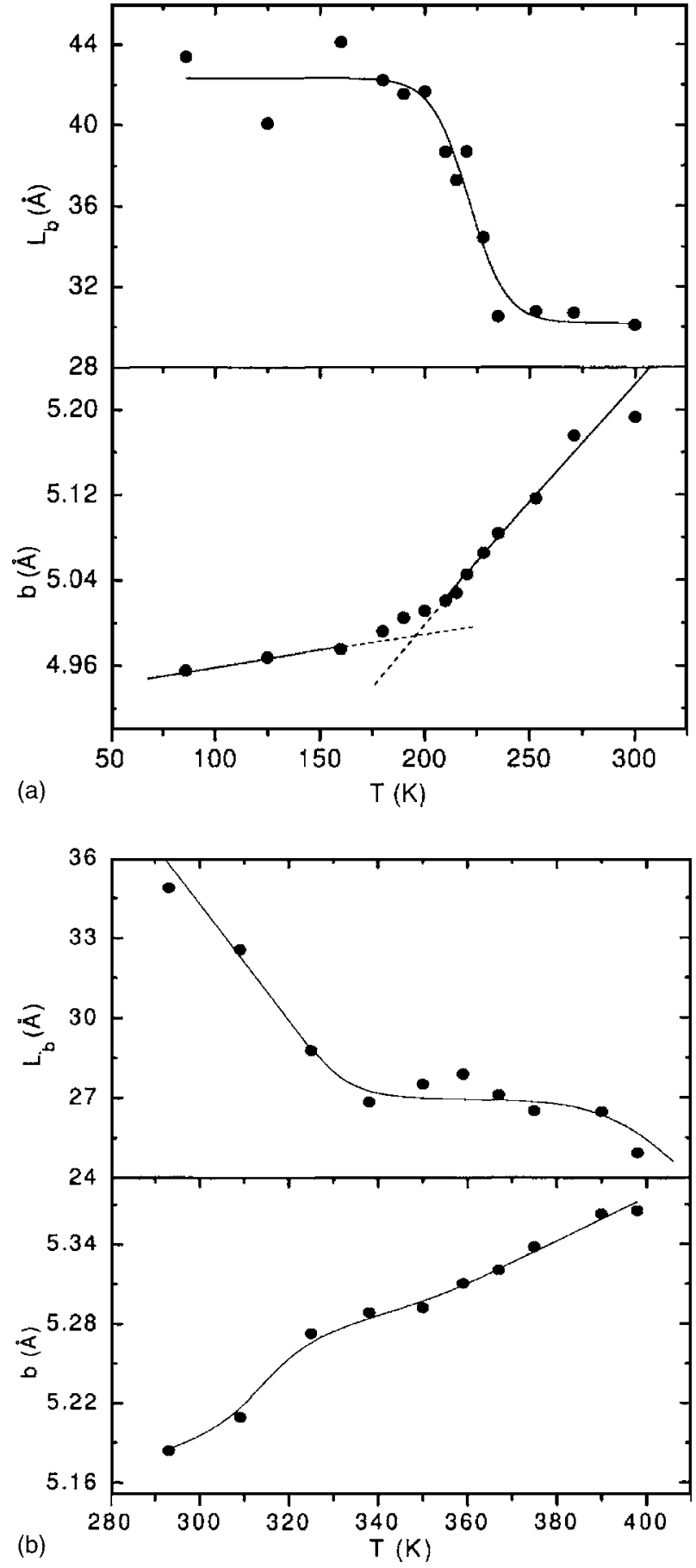

FIG. 5. The $b$ lattice constant and coherence length $L_{b}$ as function of temperature for the 0.96-layer deuterated squalane film adsorbed on Grafoil: (a) below room temperature and (b) above room temperature. Error bars are not shown but are commensurate with the indicated scatter in the data points.

dered structure; for $\mathrm{C} 24$, we concluded that there was a transition from a crystalline to a smectic phase. ${ }^{17}$ A similar transition also seems to occur in the squalane monolayer. We see in Fig. 5(a) a change in the slope of the lattice parameter versus temperature curve at around $200 \mathrm{~K}$. There is also a large decrease in the monolayer coherence length in the temperature range of $200-230 \mathrm{~K}$. We interpret this behavior as indicating a transition from a crystalline phase with shortrange translational order to a smectic phase at $\sim 210 \mathrm{~K}$.

Third, we find a smaller coherence length of the 


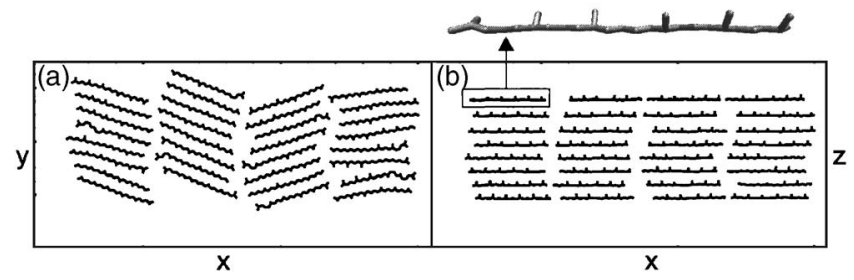

FIG. 6. (a) Top view of the simulation cell for the squalane monolayer after equilibration at $86 \mathrm{~K}$. (b) Side view of the individual molecules in the snapshot in (a). The molecules in (b) have been displaced along $z$ (normal to the surface) in order to be able to distinguish them; for example, the fifth molecule from the top in the second column of molecules in (b) is identical to the fifth molecule from the top in the second column in (a).

squalane monolayer at each temperature compared to a C24 monolayer. ${ }^{17}$ This clearly indicates that there is less translational order than in the $\mathrm{C} 24$ film.

The MD simulations have been used to investigate the reasons for the observed differences and similarities in structure between the two monolayers. We have performed simulations at a series of temperatures beginning at $86 \mathrm{~K}$ and calculated the structure factor of the squalane system for direct comparison with the observed diffraction patterns.

We begin by discussing the reason for the squalane system having less translational order than the $\mathrm{C} 24$ monolayer. We find that the squalane molecule becomes distorted from its ground state conformation when adsorbed on the graphite surface. This is evident from the snapshot of the simulation cell at low temperature. In Fig. 6(a), we see a top-down view of the equilibrated system at $86 \mathrm{~K}$ where the molecules are arranged in lamellae. Except for a few gauche defects at the ends of the chains, all of the 21 dihedral torsion angles correspond to a trans conformation. The distortion of the molecules becomes clear in a side view, as shown in Fig. 6(b). To make it possible to distinguish all molecules, we have displaced them along the ordinate axis; i.e., the molecule at the top in the first column of molecules in Fig. 6(b) is identical to the molecule at the corresponding position in Fig. 6(a) and so on. From Fig. 1(b), it is clear that three of the methyl side groups point downwards towards the surface when the molecule is in its ground state with the plane of the carbon skeleton parallel to the surface; however, in none of the molecules in Fig. 6(b) do we see a methyl group pointing downwards. Thus, when the molecules adsorb on the graphite surface, they are distorted mainly by changes in the dihedral torsion angles to make all methyl groups point away from the surface. In addition, for $\mathrm{C} 24$, we found that the carbon atoms were at an average distance of $3.56 \AA$ above the surface; based on the ground state molecular configuration in Fig. 1, we had expected to find the plane of the carbon backbone in squalane at a height of about $4.3 \AA$ above the surface. However, the simulations showed after equilibration that the height of the squalane molecule was about $3.92 \AA$. These features indicate sufficient flexibility in the torsion angles for the molecule-substrate interaction to distort the squalane molecule. We suggest that this distortion affects the close packing of the molecules, reducing the coherence length of the crystalline monolayer as observed in the experiments.

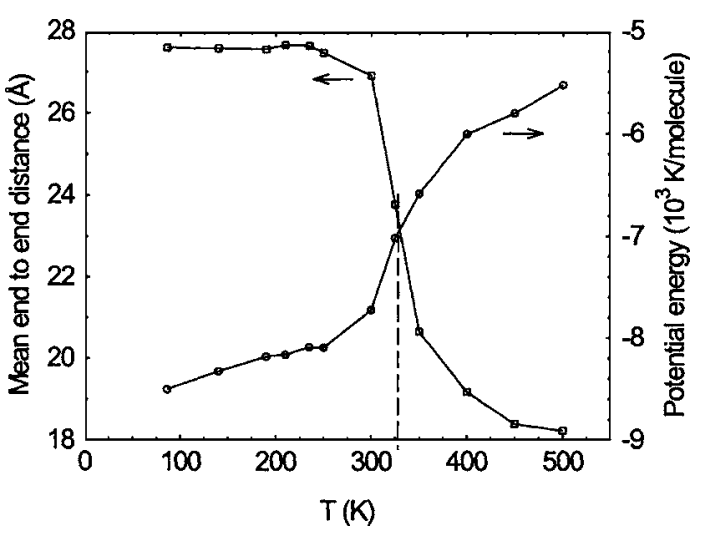

FIG. 7. Average intermolecular potential energy and end-to-end distance of the molecules as function of temperature as calculated from the MD simulations of a squalane monolayer. No quantitative uncertainty analysis was performed, but the calculated data points are based on MD simulation results with a standard deviation of the total energy on the $1 \%$ level.

In simulations of a $\mathrm{C} 24$ monolayer we have not been able to reproduce the crystalline-to-smectic phase transition observed at about $215 \mathrm{~K}$. Since the neutron diffraction patterns indicate a transition from a structure that is incommensurate with the graphite surface to one which is commensurate upon cooling, it is reasonable to assume that the corrugation in the holding potential drives the transition. We concluded that the united atom model of the molecules is inadequate for simulating this transition since it may underestimate the corrugation of the potential. An all-atom model may be better able to simulate the transition, since the methylene and methyl hydrogen atoms fit almost perfectly into the hexagons of carbon atoms on the graphite basal-plane surface. ${ }^{17,22}$ This should enhance the effect of the corrugation in the potential considerably.

We have also been unable to simulate a crystalline-tosmectic transition in the squalane monolayer, although in this case the crystalline phase inferred from the diffraction pattern is incommensurate with the graphite surface. The simulations yield some features that may be related to such a transition. For example, when we look at the average potential energy and average end-to-end distance of the molecules in Fig. 7, there are some very weak features that may be related to an incipient transition around $220 \mathrm{~K}$. There is also a much more dramatic feature in the molecular structure around that temperature when we examine the dihedral torsion angles associated with the methyl groups $\mathrm{S} 1-\mathrm{S} 6$. The plots in Fig. 8 show the percentage of each of the dihedral torsion angles that have a left or a right gauche form for four different temperatures. We note that at each temperature all 21 dihedral torsion angles along the chains are in the trans conformation except for a few gauche bonds near the chain ends, whereas the dihedral torsion angles associated with the S1-S6 methyl groups exhibit a different behavior. At $86 \mathrm{~K}$, we see that for each pair of torsion angles associated with one of the side groups $\mathrm{S} 2-\mathrm{S} 5$, one angle is in a left form and the other in a right form. The torsion angles associated with the end side groups S1 and S6 are both in the right form. At $210 \mathrm{~K}$, the picture is much the same, but at $235 \mathrm{~K}$, it changes dramatically. There is now a much different ratio of the left/ right gauche forms associated with the side groups. Further 

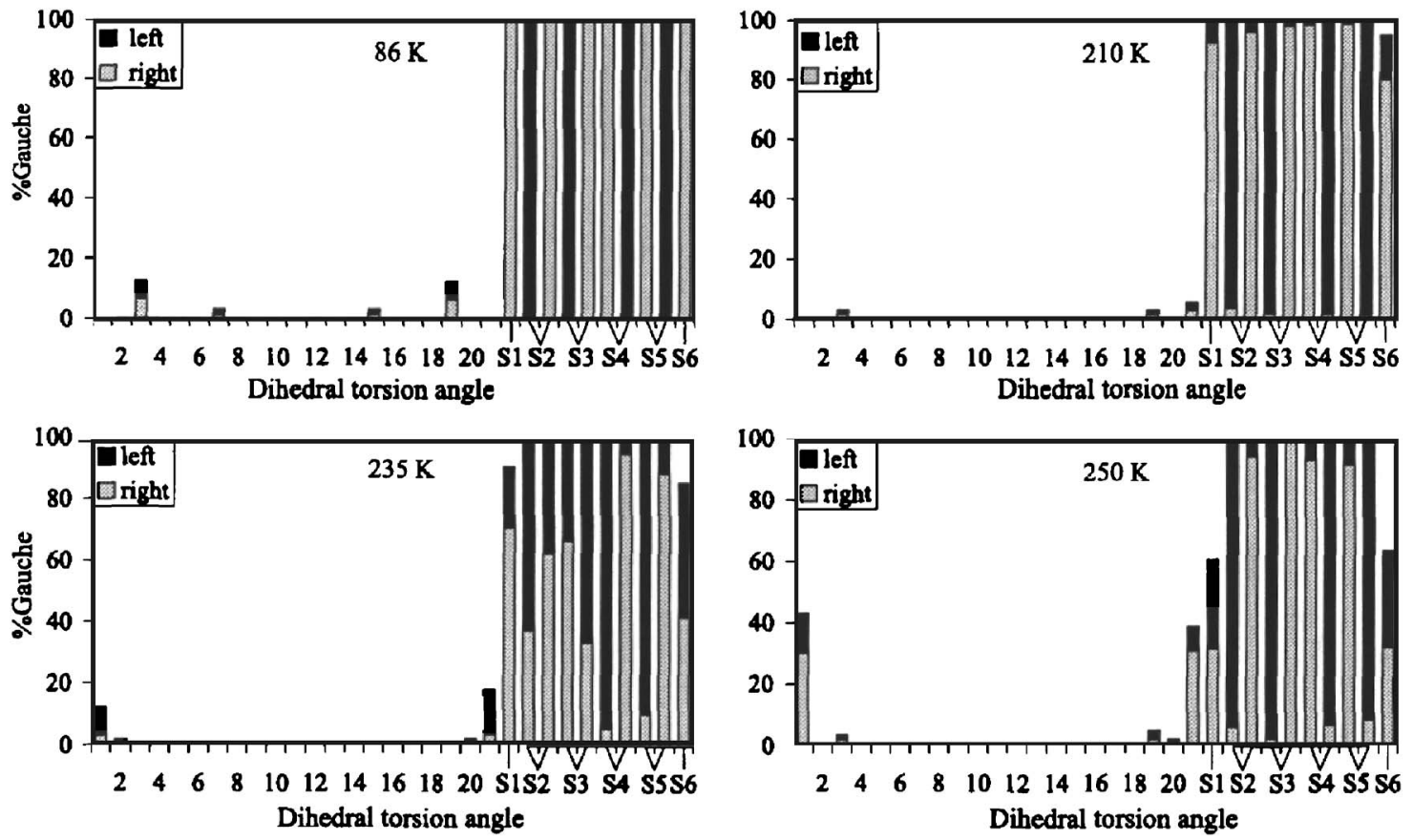

FIG. 8. The percentage of the dihedral torsion angles that are in a left or a right gauche conformation (see text) at various temperatures as calculated from the MD simulation of a squalane monolayer.

heating to $250 \mathrm{~K}$ nearly restores the left/right ratio of the gauche forms to what it was at low temperature.

As in the C24 monolayer simulations, we characterize the low-temperature phase in the MD simulations of the squalane monolayer as "smecticlike." As can be seen from the snapshots in Fig. 6(a) at $86 \mathrm{~K}$ and in Fig. 9 at $300 \mathrm{~K}$, the molecules arrange in lamellae but with translational disorder within the lamellae that increases with heating. This picture is supported by the velocity autocorrelation functions $V_{x}, V_{y}$, $V_{z}$, for motion of the molecules in the $x, y$, and $z$ directions, respectively. They are shown in Fig. 10 at a temperature of $215 \mathrm{~K}$ just above the crystalline-to-smectic transition temperature inferred by neutron diffraction (see Fig. 5) and at $260 \mathrm{~K}$ well into the smectic phase. In the $z$ direction, the molecules perform a bouncing motion on the surface, as indicated by the damped oscillatory behavior of the velocity correlation function. In the $y$ direction, the molecules bounce toward and away from each other, resulting in an oscillatory behavior that is more strongly damped than that in the $z$ direction. The motion along $x$ differs from the other two

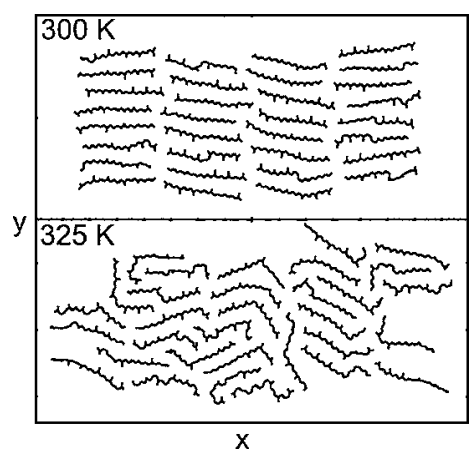

FIG. 9. Top view of the squalane monolayer simulation cell at $300 \mathrm{~K}, 25 \mathrm{~K}$ below the melting point, and at $325 \mathrm{~K}$, the melting point. directions and is consistent with a sliding motion of the molecules parallel to their long axis. It looks similar to a diffusive motion with an exponential decay of the velocity correlation function at small times. Only at longer times at $215 \mathrm{~K}$ do we see the effect of a restoring force that causes an oscillatory behavior to begin. Had the molecules been in a crystalline phase, the correlation functions along $x$ and $y$ would have been similar.

\section{B. High temperature}

Both neutron diffraction measurements and MD simulations of a C24 monolayer are consistent with a transition from a smecticlike phase to an isotropic fluid phase at about $340 \mathrm{~K} \cdot{ }^{15,17}$ It was more difficult to locate such a transition for the squalane monolayer because of the broad diffraction peaks observed in the smectic phase, as shown in Fig. 4(b). Our MD simulations are more sensitive to the smectic-tofluid transition. As we did for the C24 monolayer, we used the intermolecular potential energy and the end-to-end distance of the molecules to locate the transition temperature. This is shown in Fig. 7. There is a steep increase in the potential energy at around $325-330 \mathrm{~K}$ and an abrupt drop in the end-to-end distance of the molecules. This conclusion is confirmed by the snapshots of the simulation cell in Fig. 9. We see that the system still has the lamellar structure at $300 \mathrm{~K}$, characteristic of the smectic phase, whereas the lamellae have disappeared at $325 \mathrm{~K}$, consistent with an isotropic fluid.

When we look at the neutron diffraction patterns of the squalane monolayer in Fig. 5, we see a small kink in the $b$ lattice constant in the temperature range of $325-330 \mathrm{~K}$. Comparing with the results of the MD simulations, we may interpret this behavior as the melting signature of the squalane smectic monolayer phase. The uncertainties in the 

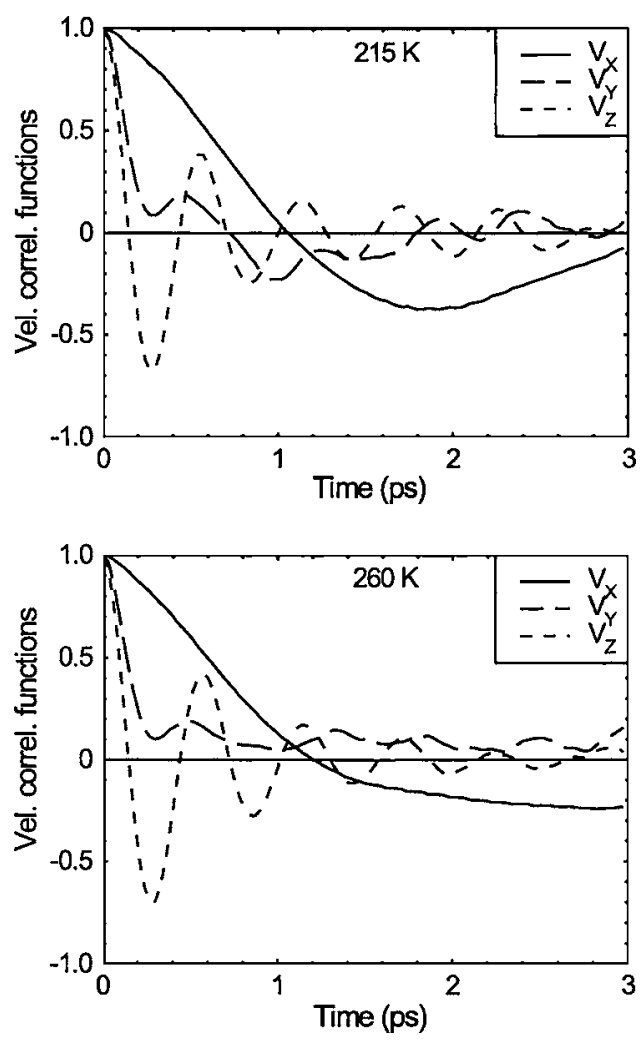

FIG. 10. Center-of-mass velocity correlation functions calculated from the MD simulations of a squalane monolayer for motion in the $x$ direction, $V_{x}$ (parallel to the long axis of the molecules), in the $y$ direction, $V_{y}$ (perpendicular to the long axis of the molecules), and in the $z$ direction, $V_{z}$ (normal to the surface for the bouncing mode of the molecules). No quantitative uncertainty analysis was performed, but the calculated data points are based on MD simulation results with a standard deviation of the total energy on the $1 \%$ level.

widths of the diffraction peaks make it difficult to extract more information about the melting transition. We note, though, that the MD simulations accurately predicted the melting temperature for the $\mathrm{C} 24$ monolayer. ${ }^{15,17}$

We also calculated the structure factors for the squalane monolayer as a function of temperature for direct comparison with the neutron diffraction patterns. The 2D powder averaged structure factors are shown in Fig. 11 in the same wave vector transfer range as in the experiments, and they are seen to be similar to the experimental patterns.

The abrupt drop in the end-to-end distance of the squalane molecule at the melting temperature as found in simulations of a C24 monolayer ${ }^{17}$ indicates a similar melting mechanism in the two monolayers as well as for dotriacontane $\left(\mathrm{C}_{32} \mathrm{H}_{64}\right) \cdot{ }^{15}$ This is further supported by the plot in Fig. 12 , where we have shown for a squalane monolayer the percentage of each of the 21 dihedral torsion angles along the chain that are in a gauche conformation as function of temperature. Below the melting transition, there are only few gauche defects. At $300 \mathrm{~K}$, i.e., $25 \mathrm{~K}$ below the squalane monolayer melting point, we see in Fig. 12 that the number of gauche defects begins to increase with most of them located at the chain ends. When the number of gauche defects reaches a sufficiently high level, the chain collapses to a more globular shape (chain melting), which drives the lattice melting. At the same time, we also observe in the simulations

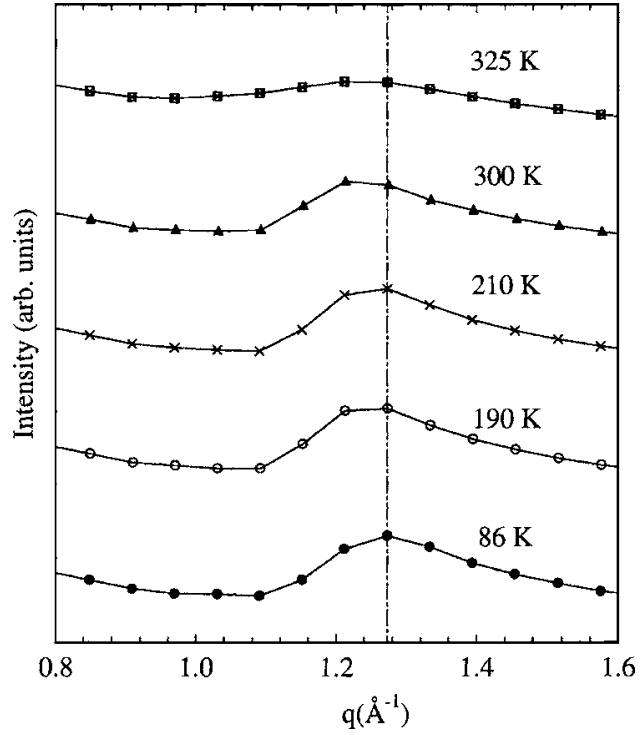

FIG. 11. The 2D powder averaged structure factor of the squalane monolayer as function of temperature as calculated from the MD simulations. No quantitative uncertainty analysis was performed, but the calculated data points are based on MD simulation results with a standard deviation of the total energy on the $1 \%$ level.

(not shown in Fig. 12) a drop in the percentage of gauche conformations for the dihedral torsion angles associated with the S1-S6 side groups. This means that several of those bonds will be in a trans conformation in the melted chains.

\section{DISCUSSION AND CONCLUSIONS}

Recently, Fuhrmann and Graham ${ }^{23}$ have used inelastic He atom scattering to determine the frequency of the molecular bouncing mode (S mode) as function of temperature for C24 and squalane adsorbed on a $\operatorname{Pt}(111)$ surface. They found a drop in the S-mode frequency over a relatively narrow temperature range, 200-250 K. Furthermore, they attributed this decrease on heating to a reduction in the molecule-substrate force constant as gauche defects are

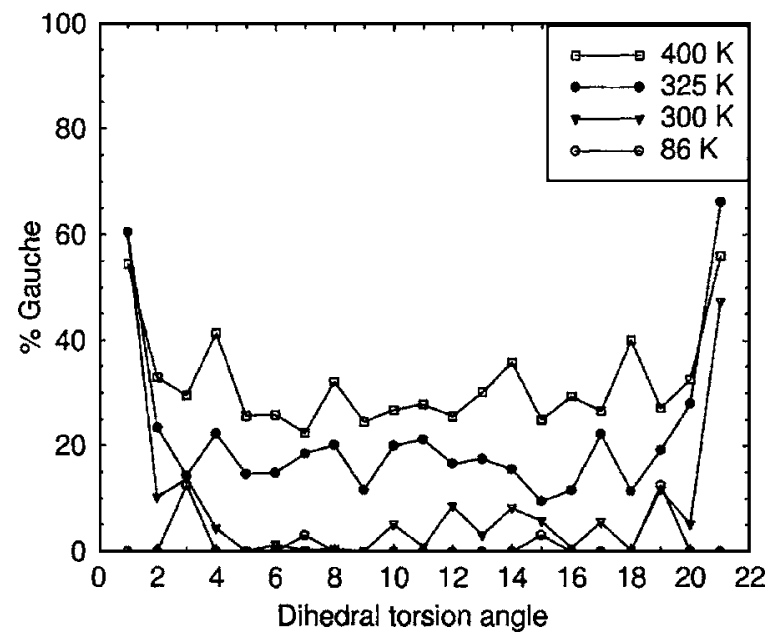

FIG. 12. The percentage of each of the 21 dihedral torsion angles along the chain that are in a gauche conformation as calculated from the MD simulations of a squalane monolayer. No quantitative uncertainty analysis was performed, but the calculated data points are based on MD simulation results with a standard deviation of the total energy on the $1 \%$ level. 
formed in a molecule and parts of it increase their height above the surface. Since the drop in frequency is smaller for squalane than that for $\mathrm{C} 24$ molecules, the authors concluded that the ends of the squalane molecules are significantly stiffer than the ends of the C24 molecules due to the molecule's methyl side groups. There is, however, no evidence of this in our simulations. The distribution of gauche defects along the backbone as a function of temperature is very similar for the two molecules (see Fig. 12 and the corresponding Fig. 4 in Ref. 15). We note that since the percentage of gauche defects depends sensitively on proximity to the melting transition, it is important to compare gauche defect distributions at the same temperatures relative to the monolayer melting point, $340 \mathrm{~K}$ (C24) and $325 \mathrm{~K}$ (squalane).

Moreover, we argue that the geometry of the squalane molecule does not allow conclusions about the stiffness of the chain ends based on measurements of the S-mode frequency. As can be seen in Fig. 2, when the dihedral torsion angles at the ends, numbered 1 and 21, change from a trans to a gauche conformation, the dihedral torsion angles associated with side groups S1 and S6 simultaneously change from gauche to trans. In other words, the S1 and S6 methyl side groups now become terminal methyl groups, while terminal groups become side groups. Thus, the initial and final molecular conformations are indistinguishable in a measurement of the S-mode frequency. This is also evident in Fig. 8 where we see that the percentage of gauche dihedral torsion angles at the ends of the molecules sums to $100 \%$; e.g., at $250 \mathrm{~K}, 60 \%$ of the dihedral torsion angles associated with side group S1 are in the gauche conformation (a decrease in $40 \%$ from the initial state) and $40 \%$ of the terminal methyl groups are gauche (an increase in $40 \%$ from the initial state). A more detailed understanding of the helium scattering experiments will require MD simulations of both molecules adsorbed on the $\operatorname{Pt}(111)$ surface rather than the graphite substrate considered here.

Our neutron diffraction experiments are consistent with monolayers of both squalane and C24 molecules forming a crystalline structure at low temperatures with a two-molecule rectangular-centered unit cell. Both films undergo a phase transition from the crystalline phase to a smectic crystalline phase at nearly the same temperature, $\sim 215 \mathrm{~K}$. In the smectic phase, the molecules are arranged in lamellae and have a common orientation but with some translational disorder within the lamellae. Our MD simulations do not reproduce this transition in the squalane monolayer except for some conformational changes associated with the side groups just below its melting point, which may indicate an incipient phase transition. We suggest that the corrugation in the holding potential may play a role both in this transition and in the distortion of the squalane molecule. Thus, it would be desirable to investigate the monolayer structure more carefully in an all-atom simulation that may give a better representation of the corrugation in the holding potential.

It is significant that at all temperatures the width of the diffraction peaks observed from the squalane monolayer is larger than that for the $\mathrm{C} 24$ monolayer, implying that the squalane monolayer has a shorter-range translational order. The MD simulations provide an explanation for this. Upon adsorption on graphite, the interaction with the basal-plane surface is strong enough to distort the molecule from its ground state by internal rotations such that all six methyl side groups point away from the surface. Without this distortion, three of the methyl groups would point down towards the surface, causing the height of the molecule to increase. The molecular distortion, despite decreasing their height, does not allow the molecules to pack as easily. This results in a shorter coherence length in the monolayer and consequently broader diffraction peaks. A calculation of the 2D averaged structure factor from the squalane monolayer simulations shows a great similarity to the observed diffraction patterns.

Both neutron diffraction measurements and MD simulations have shown that the smectic monolayer phase of C24 melts at $340 \mathrm{~K} .{ }^{17}$ Because of its broad diffraction peaks, it was difficult to determine the smectic-to-fluid transition temperature for the squalane monolayer from the diffraction data alone; however, the MD simulations clearly showed that the structure melts at about $325 \mathrm{~K}$. At that temperature, there is a small step in the measured $b$ lattice constant that may be interpreted as the signature of melting; however, without the MD results, it would be difficult to interpret this feature. There also seems to be a change in the temperature dependence of the monolayer coherence length near $325 \mathrm{~K}$; however, again it is difficult to use this behavior to locate the monolayer melting point.

Our MD simulations show the same "footprint reduction" mechanism for the melting of the squalane and C24 monolayers. ${ }^{15,24}$ The introduction of gauche defects near the center of the molecules results in their "chain melting." This gives the molecules a more globular shape and reduces their footprint on the surface. In this way, vacancies are created within the monolayers, thereby driving the lattice melting.

The almost identical melting points of the smectic phases for $\mathrm{C} 24(340 \mathrm{~K})$ and squalane $(325 \mathrm{~K})$ monolayers implies a much greater role of the surface in stabilizing the squalane monolayer because its bulk melting point is only $234 \mathrm{~K}$ compared with $322 \mathrm{~K}$ for $\mathrm{C} 24$. As discussed in the Introduction, the lower bulk melting point of squalane makes it a better lubricant at room temperature. However, this large difference in melting temperatures does not occur for adsorbed monolayers. ${ }^{15,24}$ Thus, we do not expect any significant difference in their lubrication properties in situations where molecularly thin layers are involved.

\section{ACKNOWLEDGMENTS}

This work was supported by the U.S. National Science Foundation under Grant Nos. DMR-0109057 and DMR0411748 and by the U.S. Department of Energy through Grant No. DE-FG02-01ER45912. One of the authors (A.D.E.) thanks the Oticon Foundation, Denmark, for financial support.

\footnotetext{
${ }^{1}$ J. Denis, J. Synth. Lub. 1, 210 (1984).

${ }^{2}$ J. D. Moore, S. T. Cui, H. D. Cochran, and P. T. Crummings, J. Chem. Phys. 113, 8833 (2000).

${ }^{3}$ B. N. J. Persson and F. Mugele, J. Phys.: Condens. Matter 16, R295 (2004).
} 
${ }^{4}$ S. A. Gupta, H. D. Cochran, and P. T. Cummings, J. Chem. Phys. 107, 10316 (1997).

${ }^{5}$ S. A. Gupta, H. D. Cochran, and P. T. Cummings, J. Chem. Phys. 107, 10327 (1997).

${ }^{6}$ S. A. Gupta, H. D. Cochran, and P. T. Cummings, J. Chem. Phys. 107, 10335 (1997).

${ }^{7}$ S. T. Cui, P. T. Cummings, and H. D. Cochran, J. Chem. Phys. 114, 6464 (2001).

${ }^{8}$ S. T. Cui, C. McCabe, P. T. Cummings, and H. D. Cochran, J. Chem. Phys. 118, 8941 (2003).

${ }^{9}$ H. Mo, H. Taub, U. G. Volkmann, M. Pino, S. N. Ehrlich, F. Y. Hansen, E. Lu, and P. Miceli, Chem. Phys. Lett. 377, 99 (2003).

${ }^{10}$ R. Radhakrishnan, K. E. Gubbins, and M. Sliwinska-Bartkowiak, J. Chem. Phys. 116, 1147 (2002).

${ }^{11}$ A. D. Enevoldsen, F. Y. Hansen, A. Diama, H. Taub, R. M. Dimeo, D. A. Neumann, and J. R. D. Copley, J. Chem. Phys. 126, 104704 (2007), following paper.

${ }^{12}$ K. W. Herwig, B. Matthies, and H. Taub, in Neutron Scattering in Materials Science, Materials Research Society Symposium Proceedings Vol 376, edited by D. A. Neumann, T. P. Russell, and B. J. Wuensch (Mate- rials Research Society, Pittsburgh, PA, 1994), p. 757.

${ }^{13}$ B. Matthies, Ph.D. thesis, University of Missouri-Columbia, 1999.

${ }^{14}$ A. Diama, Ph.D. thesis, University of Missouri-Columbia, 2006.

${ }^{15}$ F. Y. Hansen, K. W. Herwig, B. Matthies, and H. Taub, Phys. Rev. Lett. 83, 2362 (1999).

${ }^{16}$ D. Fuhrmann, A. P. Graham, L. Criswell, H. Mo, B. Matthies, K. W. Herwig, and H. Taub, Surf. Sci. 482-485, 77 (2001).

${ }^{17}$ B. Matthies, K. W. Herwig, L. Criswell, A. Diama, H. Mo, H. Taub, and F. Y. Hansen (in preparation for publication).

${ }^{18}$ F. Y. Hansen and H. Taub, Phys. Rev. Lett. 69, 652 (1992).

${ }^{19}$ F. Y. Hansen, J. C. Newton, and H. Taub, J. Chem. Phys. 98, 4128 (1993).

${ }^{20}$ K. W. Herwig, Z. Wu, P. Dai, H. Taub, and F. Y. Hansen, J. Chem. Phys. 107, 5186 (1997).

${ }^{21}$ E. Velasco and G. H. Peters, J. Chem. Phys. 102, 1098 (1995).

${ }^{22}$ A. J. Groszek, Proc. R. Soc. London, Ser. A 314, 473 (1970).

${ }^{23}$ D. Fuhrmann and A. P. Graham, J. Chem. Phys. 120, 2439 (2004).

${ }^{24}$ F. Y. Hansen, L. Criswell, D. Fuhrmann, K. W. Herwig, A. Diama, R. M. Dimeo, D. A. Neumann, U. G. Volkmann, and H. Taub, Phys. Rev. Lett. 92, 461031 (2004) 